\section{ANATEST: A program to generate geometric analogy problems varying in number of elements and number of transformations}

\author{
JAMES ONKEN and WILLIAM REVELLE \\ Northwestern University, Evanston, Illinois
}

Several recent studies have attempted to identify the information-processing components involved in analogical reasoning. Some have focused specifically on geometric analogies, in which terms of the analogy are geometric shapes that can be related to one another by their degrees of rotation, the number of shapes in each term, the number of sides forming each shape, and other properties of the figures. Under current informationprocessing models of analogical reasoning, each analogy term is seen as having defining characteristics or attribute dimensions, and each takes on specific values along these dimensions. For example, in the verbal analogy FATHER:SON::MOTHER..., FATHER can be represented as a set of attribute values, \{male, parent, older\}, along the dimensions of gender, familial status, and relative age. Similarly, SON $=$ \{male, child, younger $\}$.

Using this model of analogy structure, Sternberg (1977) generated models of analogical reasoning that include processes of inference, mapping, and application. Inference is the discovery process that determines the dimensions along which the first two terms of the analogy differ, and the transformations by which the second term is produced from the first. Mapping refers to the process by which relationships between the first and third terms are established. Application is a deductive process in which the relationships inferred between the first two terms of the analogy are applied to the third term to complete the analogy.

The processes of inference, mapping, and application have been used to form the structure of a package of computer programs, written in UCSD Pascal, that will produce geometric analogy problems similar to those used in psychometric tests of intelligence. ANATEST generates geometric analogies, saves the test on disk for presentation at a later time, presents previously created tests on a CRT, and records responses and response latencies. Two versions of ANATEST exist. One creates multiple-choice analogies; the second creates true-false problems.

Input. ANATEST allows an experimenter to design analogy problems with specific characteristics. Specifiable parameters of the analogy stem include: (1) num-

This study was supported, in part, by Grant MH29209 provided to W. R. by the National Institute of Mental Health. The authors' mailing address is: Department of Psychology, Nor thwestern University, Evanston, IL 60201. ber of elements-the number of geometric shapes in each term; (2) number of elements transformed-the number of elements in the first term to be transformed when creating the second term;(3) number of transformations per element; (4) number of elements mapped-the number of elements in the first term to be "mapped" when creating the third term; and (5) mappings per element. When creating test items, the specific geometric shapes used and the transformations applied to these shapes are chosen randomly from a set of eight geometric shapes and four possible transformations.

The program also allows the experimenter to specify the nature of the incorrect response alternatives (distractors). In the multiple-choice version, three distractors, in addition to the correct alternative, are created for each problem. Three of these four response altematives are selected randomly for presentation in the bottom half of the CRT. In the true-false version of ANATEST, the experimenter can specify whether an item is to be true or false. For false analogies, a single distractor is generated and presented with the analogy stem.

There are two parameters to be specified for each distractor: whether the distractor is to be created by the failure to complete all the appropriate transformations of the third analogy term (an "omissive" error) or whether an additional transformation is to be added to the correct response altemative (a "commissive" error); and the number of these omissions or commissions.

The experimenter need not specify the design of every item on a test. For each set of parameters specified (for each item "type"), the experimenter is asked to indicate the number of items of this type within each block of items and the number of item blocks within the test. Practice trials, if used, are constructed in the same fashion as test trials and are saved as a separate file. Several options that govern the presentation format of the practice and test trials are available. If desired, presentation order may be randomized within blocks. Feedback may also be presented. If this option is selected, the frequency with which feedback is provided is also specifiable.

Output. At the test-construction stage, ANATEST creates two disk files. One file contains descriptions of each test item (the parameter values set by the experimenter), and a second file contains the test items themselves (the specific attribute values of each item generated by the program).

When a test has been constructed, ANATEST is used to present the items on a CRT. Written instructions can be presented to the testtaker before the practice or test trials begin. These instructions include an introduction to geometric analogies, illustrations of eight shapes used in the analogies, and demonstrations of the four possible transformations. The presentation of 
instructions is optional for those cases in which experienced subjects are used or instructions are otherwise undesirable.

When a test has been completed, a data file is written that includes: (1) the subject's identification number; (2) the number of practice trials presented (if any); (3) response information for practice items, including solution latencies and chosen responses, the correct responses for each item, and item scores; (4) the number of test items presented; and (5) response information for the test items. Timing is done using an "external" procedure written in 6502 assembly language, but can be done using a clock, if one is available.

Program Language and Requirements. The programs that ANATEST comprises are written in Apple Pascal using UCSD Pascal P-system. ANATEST was designed for use with an Apple II equipped with $64 \mathrm{~K}$ memory and one disk drive.

Availability. Source and object code for both the multiple-choice and true-false versions of ANATEST are available from the authors. Requests should indicate the version desired and should be accompanied by $\$ 25.00$ for each version requested. Send requests to James Onken, Department of Psychology, Northwestern University, Evanston, Illinois 60201.

\section{REFERENCES}

StERnBerg, R. J. (1977). Intelligence, information processing and analogical reasoning: The componential analysis of human abilities. Hillsdale, $\mathrm{NJ}$ : Erlbaum.

(Revision accepted for publication May 11, 1984.) 